\title{
Produção e caracterização de espumas vitrocristalinas a partir de resíduos sólidos
}

\section{Production and characterization of vitrocrystalline foams from solid wastes}

\author{
Luyza Bortolotto Teixeira ${ }^{1}$, Bianca Goulart de Oliveira Maia ${ }^{1}$, Sabrina Arcaro ${ }^{1}$, \\ Noeli Sellin ${ }^{2}$, Antonio Pedro Novaes de Oliveira ${ }^{1}$
}

\author{
${ }^{1}$ Programa de Pós-Graduação em Ciência e Engenharia de Materiais (PGMAT), Laboratório de Materiais \\ Vitrocerâmicos (VIROCER), Universidade Federal de Santa Catarina ( UFSC), Campus Universitário - Trindade, \\ Caixa Postal 476, 88040-900 Florianópolis, SC. \\ e-mail: bianca.maia@outlook.com \\ ${ }^{2}$ Mestrado em Engenharia de Processos, Universidade da Região de Joinville - UNIVILLE, Rua Paulo \\ Malschitzki,10, Zona Industrial, CEP 89219 710, Joinville/SC. \\ e-mail: nsellin@yahoo.com.br
}

\section{RESUMO}

Neste trabalho, foram produzidas espumas vitrocristalinas a partir de vidros de garrafas descartadas e casca de arroz (3 a 25\% em massa), como agente porogênico. Diferentes composições foram preparadas de maneira a se obter materiais com porosidade controlada para aplicações, nas quais o isolamento térmico e a não-inflamabilidade são os principais requisitos técnicos. Os pós de cada matéria-prima (resíduos) foram homogeneizados e as misturas foram então prensadas uniaxialmente (20 MPa). Os compactos obtidos foram queimados em diferentes temperaturas $\left(700-950{ }^{\circ} \mathrm{C}\right)$ e tempos de patamares (30 - $120 \mathrm{~min})$. As matérias-primas e as espumas vitrocristalinas produzidas foram caracterizadas de acordo com suas propriedades químicas, físicas e estruturais. Os resultados mostraram que é possível produzir espumas vitrocristalinas a partir de composições otimizadas com $85 \%$ de vidros de garrafas e $15 \%$ de casca de arroz, queimadas a $900{ }^{\circ} \mathrm{C}$ por diferentes tempos. Tais espumas vitrocristalinas apresentaram porosidade variando entre 80 e $84 \%$ enquanto a condutividade térmica e a resistência mecânica à compressão variaram entre 0,068 e $0,082 \mathrm{~W} / \mathrm{m} . \mathrm{K}$ e entre 1,1 e 1,8 MPa, respectivamente.

Palavras-chave: Espumas vitrocristalinas. Casca de arroz. Isolantes térmicos. Resíduos sólidos.

\section{ABSTRACT}

Vitrocrystalline foams from discarded glass bottles and rice husk (3 to $25 \mathrm{wt} \%$ ) as pore forming agent were produced in this work. Different compositions were prepared in order to obtain materials with controlled porosity in applications where thermal insulation and non-flammability are major requirements. Well-homogenized powders of the formulated compositions were uniaxially pressed (20 $\mathrm{MPa})$ and the obtained compacts fired at different temperatures $\left(700-950^{\circ} \mathrm{C}\right)$ and holding times $(30-120$ min). Raw materials and the obtained vitrocrystalline foams were characterized according to their chemical, physical and structural properties. The results showed that it is possible to produce vitrocrystalline foams from optimized compositions containing $85 \%$ glass bottle and $15 \%$ rice husk, fired at $900^{\circ} \mathrm{C}$ for different holding times. These vitrocrystalline foams presented porosity varied from 80 to $84 \%$ while the thermal conductivity and compressive strength varied between 0.068 and $0.082 \mathrm{~W} / \mathrm{m} . \mathrm{K}$ and from 1.1 to $1.8 \mathrm{MPa}$, respectively.

Keywords: Vitrocrystalline foams. Rice husk. Thermal insulators. Solid wastes.

\section{INTRODUÇÃO}

Espumas vítreas são materiais extremamente porosos (com porosidades tipicamente superiores a $70 \%$ ), não inflamáveis, com resistências química e mecânica (resistência mecânica à compressão entre 0,4 e 6 
MPa) adequadas e com baixa condutividade térmica $(0,04$ a $0,08 \mathrm{~W} / \mathrm{m} . \mathrm{K})$ [1,2]. A porosidade pode ser classificada como aberta, fechada ou mista e, com relação ao seu tamanho, pode ser micro (menor que 2 $\mathrm{nm}$ ), meso (2 a $50 \mathrm{~nm}$ ) ou contendo macroporos (acima de $50 \mathrm{~nm}$ ) [2,3]. As espumas vítreas, quando usadas como blocos estruturais, por exemplo, são fáceis de manusear e possuem excelente compatibilidade com o concreto, facilitando e agilizando a construção, com baixos custos de transporte. Esta combinação de propriedades faz com que tais materiais sejam muito interessantes na indústria da construção (tal como para o isolamento de tetos, paredes, pisos, lareiras e churrasqueiras), em aplicações nas quais a temperatura não seja superior a $500{ }^{\circ} \mathrm{C}$ [2].

Os principais fatores que determinam suas aplicações são a morfologia das células, a distribuição e interconectividade da porosidade. Dentre os materiais normalmente utilizados para a obtenção de materiais porosos, pode-se mencionar a alumina, mulita, carbeto de silício, zircônia e hidroxiapatita e também materiais vítreos ou parcialmente cristalinos, como os vidros e os vitrocerâmicos $[2,4,5,6]$. Em tais casos, o uso de resíduos provenientes da reciclagem de vidro em geral ou de indústrias cerâmicas é uma possibilidade a ser explorada, pois a reciclagem e reutilização do vidro contribuem para a matriz energética nacional com a economia de grande quantidade de energia. De fato, para a obtenção de vidros a partir de matérias-primas (originais) naturais e/ou sintéticas são necessários $4.500 \mathrm{~kJ} / \mathrm{kg}$ de vidro produzido, enquanto que para a obtenção de produtos de vidro a partir da sua reciclagem necessita-se somente $500 \mathrm{~kJ} / \mathrm{kg}$ de vidro produzido. Os resíduos da indústria do vidro e de embalagens de vidro usadas no dia-a-dia são descartados com o lixo doméstico, o que representa $2 \%$ de todo o resíduo sólido urbano da cidade de São Paulo (equivalente a um descarte de 7.000 t/mês de vidro) [7]. Quando a espuma resultante apresenta uma quantidade considerável de fase cristalina, utiliza-se a denominação espuma vitrocristalina para denominar tais materiais [2].

Várias técnicas para a produção de materiais porosos são utilizadas e as etapas do processo geralmente compreendem a preparação de uma suspensão de pó cerâmico, conformação e tratamento térmico (queima). Dentre as técnicas mais conhecidas para a produção de materiais porosos pode-se mencionar os métodos da réplica polimérica, gelcasting, espumação e incorporação de agentes porogênicos [2].

A incorporação de agentes porogênicos ou agentes formadores de poros, devido à sua simplicidade, é a técnica mais utilizada na produção de espumas vítreas e vitrocristalinas, e esta consiste na incorporação de determinados materiais no corpo cerâmico, os quais são eliminados durante o processo de queima, resultando em poros cujo tamanho está associado ao das partículas destes agentes que podem ser sintéticos (como os polímeros) ou naturais (como os resíduos vegetais e minerais) [2, 6]. Os resíduos, em geral, exibem em suas composições diferentes constituintes, que podem agregar valor ao produto resultante e podem diminuir o custo de sua produção sendo um agente porogênico alternativo [1].

Diversos estudos já comprovaram a possibilidade de obtenção de espumas vítreas e vitrocristalinas a partir de vidros de garrafas descartadas, vidros de tubos de raios catódicos e lâmpadas, usados como matriz vítrea, e grafite, resíduos de estações de tratamentos de efluentes, cascas de ovos, calcita e dolomita como agentes porogênicos alternativos. Os resultados obtidos indicaram porosidades entre 40 e $90 \%$, condutividade térmica entre 0,08 e $0,175 \mathrm{~W} / \mathrm{m} . \mathrm{K}$ e resistência mecânica à compressão entre 1,5 e $20 \mathrm{MPa}[8,9,10,11]$.

Um resíduo que pode ser utilizado como agente formador de poros, devido às suas propriedades e disponibilidade, é a casca de arroz (CA). Depois do milho, o arroz é o cereal mais produzido no mundo todo. $\mathrm{O}$ arroz é cultivado em grandes quantidades em muitos países, incluindo a China, o subcontinente indiano, sudeste da Ásia e regiões da África e da América do Sul [12]. No Brasil, de acordo com dados da Companhia Nacional de Abastecimento (CONAB), a produção de arroz em casca em 2015 foi estimada em 12,5 milhões de toneladas [13].

A casca de arroz é um material fibroso composto principalmente por celulose, hemicelulose, lignina além de outra fração de compostos orgânicos. Por possuir lenta degradação, a casca permanece inalterada por um longo período de tempo, causando grande prejuízo ao meio ambiente. Sem valor comercial como resultado de sua dureza, fibrosidade e natureza abrasiva, a casca de arroz é tradicionalmente descartada em aterros, ocupando grandes volumes, ou utilizada como combustível para geração de energia na forma de calor, empregado na secagem do próprio cereal [12, 14]. O resultado desta ultima utilização/emprego no Brasil consome somente cerca de $30 \%$ da casca produzida. Considerando que cerca de $20 \%$ da massa do grão de arroz é constituída pela casca $[12,15,16]$, e $4 \%$ corresponde a cinzas, estima-se que cerca de 3 milhões de toneladas de casca de arroz são geradas no 
país.

Neste contexto, o principal objetivo deste trabalho foi produzir espumas vitrocristalinas a partir de vidros de garrafas descartadas, utilizadas como matriz vítrea, e casca de arroz, como agente porogênico. Diferentes amostras foram preparadas, por meio da mistura dos pós obtidos e compactação uniaxial e, em uma etapa posterior, a queima dos compactos, de modo a obter materiais com porosidade controlada para aplicações nas quais o isolamento térmico e a não-inflamabilidade são os principais requisitos técnicos. Esta iniciativa é mais uma alternativa para a destinação e utilização da casca de arroz a qual pode originar produtos com agregação de valor e com respeito ao meio ambiente.

\section{MATERIAIS E MÉTODOS}

As matérias-primas utilizadas na produção das espumas foram garrafas de vidro (VG) de diferentes colorações (clara, verde e marrom), empregadas como matriz vítrea e casca de arroz (CA) utilizada como agente porogênico. As composições químicas dos vidros de garrafas claras (VGC), verdes (VGV) e marrons (VGM) foram obtidas por Fluorescência de Raios X (Philips, modelo PW 2400).

A casca de arroz foi caracterizada por análise química aproximada, conforme procedimentos descritos nas normas ASTM, E871-82 [17], E872-82 [18], E1755-01 [19] e E1756-08 [20]. A umidade (U) foi determinada em amostras secas em estufa laboratorial (SP LABOR) por 360 min, verificando-se a diferença da massa da amostra antes $\left(\mathrm{m}_{\mathrm{i}}\right)$ e após secagem $\left(\mathrm{m}_{\mathrm{s}}\right)$ utilizando a Equação 1. Os teores de sólidos voláteis ( $\mathrm{SV}$, fração que primeiramente entra em combustão na queima) e teor de cinzas (TC, fração inerte) foram obtidos pela calcinação da amostra a $575{ }^{\circ} \mathrm{C}$, por $180 \mathrm{~min}$, com aquecimento a 10 ${ }^{\circ} \mathrm{C} / \mathrm{min}$, calculando-se as diferenças das massas da amostra seca e calcinada $\left(\mathrm{m}_{\mathrm{c}}\right)$, por meio das Equações 2 e 3 .

O teor de carbono fixo (TCF, fração mássica resultante da liberação dos compostos voláteis) foi determinado utilizando a Equação 4 por meio dos valores de sólidos voláteis e cinzas, anteriormente determinados conforme as Equações 2 e 3 [21, 22].

$$
\begin{aligned}
& \left.\mathrm{U}=\left[\left(\mathrm{m}_{\mathrm{i}}-\mathrm{m}_{\mathrm{s}}\right) / \mathrm{m}_{\mathrm{i}}\right)\right] \times 100 \\
& \mathrm{SV}=\left\{\left[\left(\mathrm{m}_{\mathrm{s}}-\mathrm{m}_{\mathrm{c}}\right) / \mathrm{m}_{\mathrm{i}}\right] \times 100\right\}-\mathrm{U} \\
& \mathrm{TC}=\left(\mathrm{m}_{\mathrm{c}} / \mathrm{m}_{\mathrm{s}}\right) \times 100 \\
& \mathrm{TCF}=100-(\mathrm{SV}+\mathrm{TC})
\end{aligned}
$$

Para melhor promover o processo de formação da espuma, os pós de vidro e do agente porogênico devem apresentar partículas menores que $4 \mathrm{~mm}$ [2]. Para facilitar este processo, as matériasprimas foram moídas até apresentarem-se passantes em peneira de $45 \mu \mathrm{m}$. Os vidros de garrafas foram, inicialmente, fragmentados e moídos em moinho de martelos (SERVITECH, CT-058), e os pós resultantes foram então moídos a seco por $120 \mathrm{~min}$ em moinho rápido (SERVITECH, CT-242) usando jarro e bolas de aluminas, obtendo-se tamanhos de partículas $\left(\mathrm{d}_{50}\right)$ de aproximadamente 2,0 $\mu \mathrm{m}$ (conforme analisador de tamanho de partículas Malvern, Mastersizer 2000). A casca de arroz (CA) foi moída a seco em moinho rápido (SERVITECH, CT-058) resultando em pós com tamanhos de partículas menores que $71 \mu \mathrm{m}$. Apesar do peneiramento prévio, o $\mathrm{d}_{50}$ observado para o pó da casca de arroz foi superior a $45 \mu \mathrm{m}$ e este fato é resultado do formato alongado das partículas que possuem comprimento muito maior do que sua espessura (elevada razão de aspecto).

Em uma etapa posterior, composições contendo 75 a 97\% (em massa) de vidros de garrafas (mistura dos pós em iguais proporções das três colorações de vidros de garrafas) e casca de arroz (CA) variando de 3 a 25\% (em massa) foram misturadas a úmido (40\% de sólidos) e homogeneizadas em agitador mecânico (RW 20 digital, IKA) por $120 \mathrm{~min}$ a $200 \mathrm{rpm}$ para promover uma melhor homogeneização dos pós. Testes preliminares mostraram que a mistura a seco dos pós dos resíduos não promove uma distribuição homogênea de poros nas espumas obtidas.

Subsequentemente, as suspensões obtidas foram secas em estufa laboratorial (SP LABOR®) a $110 \pm 10^{\circ} \mathrm{C}$ e homogeneizadas em moinho rápido de alumina (SERVITECH, CT-058) por 1 min e os pós obtidos foram umidificados (5\% de água). As composições preparadas foram então prensadas uniaxialmente (20 MPa) em uma matriz de aço utilizando uma prensa hidráulica (Bovenau P10 ST). 
O comportamento térmico durante a queima das matérias-primas e das composições preparadas foi analisado em um dilatômetro ótico (Expert System Solution Misura ODHT) e em um analisador térmico simultâneo ATG-DSC (TA Instruments, SDT Q600), com taxa de aquecimento de $10{ }^{\circ} \mathrm{C} / \mathrm{min} \mathrm{e}$ fluxo de ar sintético a $10 \mathrm{~cm}^{3} / \mathrm{min}$. Com base nos resultados das análises térmicas, amostras de compactos de pós foram queimados em forno de laboratório (Jung J200) em diferentes temperaturas (de 700 a $950{ }^{\circ} \mathrm{C}$ ) por diferentes patamares de tempos (de 30 a $120 \mathrm{~min}$ ), com taxa de aquecimento de $10{ }^{\circ} \mathrm{C} / \mathrm{min} \mathrm{e}$ com resfriamento natural.

A densidade real $\left(\rho_{r}\right)$ das amostras em pó foi determinada utilizando um picnômetro a hélio (AccuPyc 1340, Micromeritics, USA). A retração linear (\%) e a densidade aparente $\left(\rho_{a}\right)$ das espumas foram determinadas relacionando suas dimensões geométricas (obtidas por meio de um paquímetro, modelo Mitotoyo, resolução de 0,01 mm), e suas massas (obtidas com balança analítica Shimadzu AX200 com resolução de $0,001 \mathrm{~g}$ ). A partir das medidas geométricas e da densidade real, a porosidade $(\mathcal{E})$ das espumas vítreas obtidas foi calculada de acordo com a relação:

$$
\varepsilon(\%)=\left[1-\left(\rho_{\mathrm{a}} / \rho_{\mathrm{r}}\right)\right] \times 100
$$

A microestrutura de poros pôde ser visualizada a partir de imagens das superfícies de fratura de espumas queimadas, obtidas em microscópio eletrônico de varredura, MEV (JEOL JSM-6390LV). O tamanho de poros das espumas vítreas foi determinado por meio de um software de análise de imagem, ImageJ, a partir do método do intercepto linear (sendo realizadas em média 300 medições para cada grupo de imagens correspondente a uma condição de queima) [23]. A resistência mecânica à compressão das espumas vitrocristalinas foi determinada por meio de uma máquina universal de ensaios mecânicos (EMIC DL modelo 2000) em quatro amostras (velocidade de carregamento: $1 \mathrm{~mm} / \mathrm{min}$ ) para cada condição de queima (dimensões nominais das amostras: $30 \mathrm{~mm}$ de diâmetro e $10 \mathrm{~mm}$ de espessura). A condutividade térmica das espumas foi obtida utilizando um equipamento TCi Thermal Conductivity CTHERM TECHNOLOGIES em amostras com dimensões de $30 \mathrm{~mm}$ X $10 \mathrm{~mm}$.

Para investigar a natureza amorfa ou cristalina das espumas produzidas foi utilizado um difratômetro de raios X (Philips, modelo X'Pert) acoplado a um tubo de cobre (radiação $\mathrm{CuK} \alpha$ ), com potência de $40 \mathrm{kV}$ e $30 \mathrm{~mA}$. Para a realização das difratometrias de raios $\mathrm{X}$, foram preparadas amostras em pós e os parâmetros de análise fixados em: passo de $0,02^{\circ}$, tempo de passo de $1 \mathrm{~s}$ e intervalo de medida em $2 \theta$, de 3 a $80^{\circ}$. Para identificação das fases cristalinas formadas foi utilizado o banco de dados JCPDS.

\section{RESULTADOS}

A Tabela 1 mostra os resultados das análises químicas dos vidros de garrafas claras (VGC), verdes (VGV) e marrons (VGM). A Tabela 2 apresenta os resultados da análise química aproximada das CA utilizadas e também resultados encontrados na literatura, os quais foram obtidos por meio dos mesmos procedimentos normatizados utilizados neste trabalho.

Tabela 1: Composição química dos vidros de garrafas claras (VGC), verdes (VGV) e marrons (VGM) utilizadas neste trabalho.

\begin{tabular}{|c|c|c|c|c|c|c|c|c|c|}
\hline \multirow{2}{*}{ Vidros } & \multicolumn{10}{|c|}{ Óxidos constituintes (\%) } \\
\cline { 2 - 10 } & $\mathbf{S i O}_{\mathbf{2}}$ & $\mathbf{A l}_{\mathbf{2}} \mathbf{O}_{\mathbf{3}}$ & $\mathbf{F e}_{\mathbf{2}} \mathbf{O}_{\mathbf{3}}$ & $\mathbf{C a O}$ & $\mathbf{K}_{\mathbf{2}} \mathbf{O}$ & $\mathbf{M g O}$ & $\mathbf{N a}_{\mathbf{2}} \mathbf{O}$ & $\mathbf{P}_{\mathbf{2}} \mathbf{O}_{\mathbf{5}}$ & $\mathbf{T i O}_{\mathbf{2}}$ \\
\hline VGC & 71,2 & 2,2 & 0,1 & 9,6 & $<0,1$ & - & 16,8 & $<0,1$ & $<0,1$ \\
\hline VGV & 68,3 & 2,1 & 0,4 & 8,9 & 0,4 & 1,8 & 18,0 & $<0,1$ & 0,1 \\
\hline VGM & 67,1 & 2,0 & 0,8 & 9,5 & 0,3 & 0,6 & 19,6 & $<0,1$ & $<0,1$ \\
\hline
\end{tabular}

Tabela 2: Análise química aproximada da casca de arroz (CA).

\begin{tabular}{|c|c|c|c|c|}
\hline Referências & CA & Kok; Özgür [23] & Mhilu [24] & García et al. [20] \\
\hline Componente & \multicolumn{4}{|c|}{ Quantidade (\%) } \\
\hline Umidade (U) & $10,4 \pm 0,5$ & 2,4 & 8,8 & $7,3 \pm 0,1$ \\
\hline Sólidos Voláteis (SV) & $62,7 \pm 0,6$ & 64,1 & 59,2 & $73,0 \pm 2,0$ \\
\hline Teor de Cinzas (TC) & $18,5 \pm 1,3$ & 11,1 & 26,2 & $13,7 \pm 0,4$ \\
\hline $\begin{array}{c}\text { Teor de Carbono } \\
\text { Fixo (TCF) }\end{array}$ & $18,8 \pm 0,9$ & 22,4 & 14,6 & 13,3 \\
\hline
\end{tabular}


De acordo com os dados da Tabela 1, os vidros de garrafas são constituídos principalmente por óxidos de silício, cálcio e sódio. Além destes óxidos, pode ser observada também a presença de outros óxidos em menores quantidades e com funções específicas. A alumina em proporções variando de 2,0 a $2,2 \%$ é tipicamente encontrada em vidros sódico-cálcicos já que atua como estabilizador da estrutura vítrea e aumenta a durabilidade química [24]. Os óxidos de titânio e de ferro (principalmente) encontrados atuam como elementos cromóforos. De fato, quando a quantidade de óxido de ferro varia de 0,1 a $0,8 \%$, o vidro claro torna-se verde e marrom. Tais composições são típicas dos vidros sódicocálcicos utilizados na fabricação de vidros planos, vidros de janelas e vidros de embalagens em geral [24].

Conforme a Tabela 2, apesar de baixo, aproximadamente $10 \%$, o valor de umidade encontrado nas amostras de CA, é consideravelmente mais elevado do que aqueles valores reportados na literatura $[21,15,16]$, provavelmente devido a elevada umidade relativa média anual do ar $(85 \%)$ da região de Santa Catarina [25].

A fração inicial de CA que entra em combustão, sólido volátil, corresponde a aproximadamente $63 \%$ da casca de arroz, a qual, por sua vez, está relacionada às frações lignocelulósicas da biomassa, que são termicamente degradadas e oxidadas durante a combustão. Quanto maior o teor de sólidos voláteis em uma biomassa, maior a energia liberada durante a combustão [26, 27].

Após a combustão da casca de arroz, o material remanescente corresponde ao teor de cinzas que, para a CA analisada, foi de aproximadamente $19 \%$. Segundo bibliografia $[15,16]$, após a queima da matéria orgânica contida na casca de arroz sobra a cinza de difícil degradação e com pouquíssimos nutrientes para o solo, contendo aproximadamente 95 a $98 \%$ de sílica no estado amorfo e uma pequena fração de óxidos alcalinos (aproximadamente $2 \%$ ).

$\mathrm{O}$ teor de carbono fixo determinado pela equação 4 (relacionado à massa restante do material após a saída de compostos voláteis na queima, excluindo as cinzas e a umidade) foi de aproximadamente $19 \%$. Este valor é próximo aos valores apresentados em bibliografias, conforme verificado na Tabela 2. O carbono apresenta expansão em temperaturas entre 650 e $1000{ }^{\circ} \mathrm{C}$ [28], portanto, este valor verificado experimentalmente é significativo para auxiliar na etapa de formação da espuma.

A Figura 1 mostra curvas de retração linear do vidro de garrafa (VG) e de calorimetria exploratória diferencial (DSC) e de termogravimetria (ATG) da casca de arroz (CA).

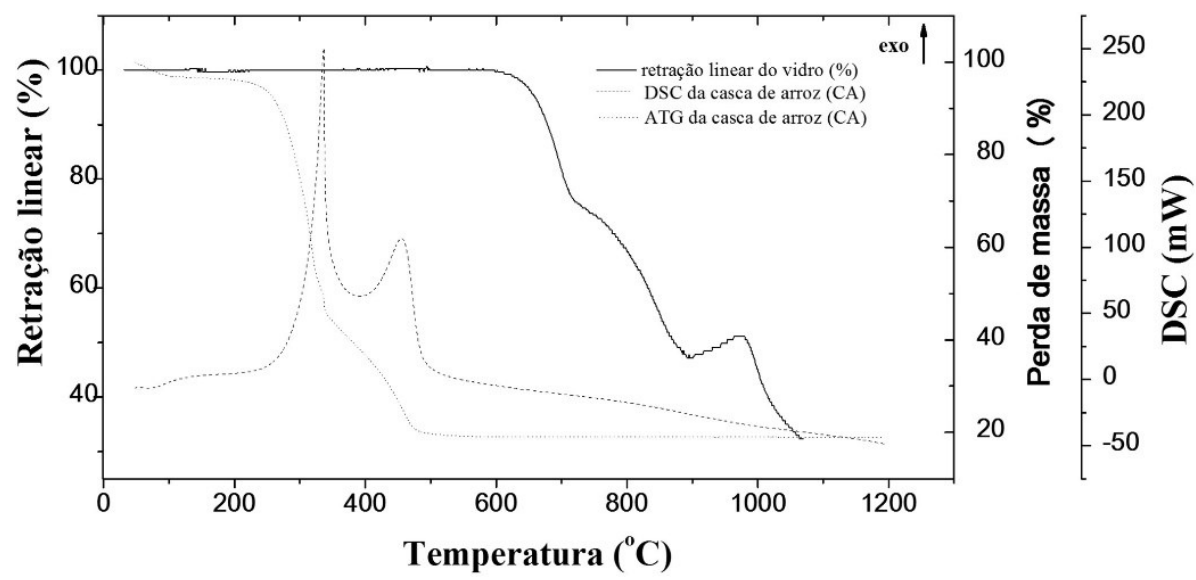

Figura 1: Curvas de retração linear do vidro de garrafa (VG) e das análises de calorimetria exploratória diferencial (DSC) e termogravimetria (ATG) da casca de arroz (CA).

É possível observar, a partir da análise da Figura 1, que a densificação (em termos de retração) do VG ocorre de maneira mais acentuada entre 600 e $700^{\circ} \mathrm{C}$ e, a uma velocidade menor até $900{ }^{\circ} \mathrm{C}$. A partir de $900{ }^{\circ} \mathrm{C}$, tem início a expansão do vidro a qual precede o início de sua fusão (a $950{ }^{\circ} \mathrm{C}$ ). $\mathrm{O}$ intervalo de temperatura mais adequado para a formação de porosidade em vidros sódico-cálcicos, de acordo com a literatura [2], está compreendido entre 800 e $1000^{\circ} \mathrm{C}$ e corresponde a viscosidades entre $10^{5}$ e $10^{3}$ Pa.s. Esta viscosidade é adequada para reter os gases originados na decomposição do agente porogênico no interior da matriz vítrea, promovendo a expansão e, consequentemente, originando poros.

Na curva de ATG da casca de arroz (Figura 1), é possível observar três estágios de degradação 
térmica. O primeiro estágio ocorre até $150{ }^{\circ} \mathrm{C}$ com uma perda mássica de aproximadamente $3,5 \%$, a qual pode ser atribuída a perda de umidade. Este resultado é significativamente diferente ao valor de umidade verificado na Tabela 2 devido ao fato de que para a análise química aproximada a amostra foi a casca de arroz in natura e para a análise térmica, a amostra ensaiada foi a casca de arroz moída e previamente seca em estufa. Nos dois casos, pode ter ocorrido a absorção da umidade do ar. O segundo estágio ocorre entre 150 e $500{ }^{\circ} \mathrm{C}$, caracterizado peladecomposição da fração orgânica, como a celulose, hemicelulose e parte da lignina, com a maior perda mássica de aproximadamente $77 \%$. Acima de $500{ }^{\circ} \mathrm{C}$ ocorre o terceiro estágio de degradação térmica, onde verifica-se a decomposição da fração restante de lignina (cerca de $4 \%$ de perda de massa).

Segundo alguns autores [26, 29] a hemicelulose é composta de vários sacarídeos (xilose, manose, glicose, galactose, etc.), com estrutura aleatória, amorfa, com várias ramificações. A decomposição dos materiais voláteis (formando $\mathrm{CO}, \mathrm{CO}_{2}$, e alguns hidrocarbonetos) ocorre facilmente a baixas temperaturas $\left(220\right.$ a $\left.315^{\circ} \mathrm{C}\right)$. Diferente da hemicelulose, a celulose é constituída por um longo polímero de glicose, sem ramificações e se decompõe em temperaturas compreendidas entre 315 e $400{ }^{\circ} \mathrm{C}$ [26]. Já a lignina é repleta de anéis aromáticos com várias ramificações. A quebra das suas ligações químicas ocorre numa faixa de temperatura extremamente ampla. Por este motivo a degradação da lignina ocorreu, neste caso, no intervalo de temperatura compreendido entre 150 e $900{ }^{\circ} \mathrm{C}$.

Na curva de DSC da CA (Figura 1) são observados dois eventos exotérmicos, com máximos em $336{ }^{\circ} \mathrm{C}$ e $455^{\circ} \mathrm{C}$, os quais correspondem à degradação da fração orgânica, celulose, hemicelulose e parte da lignina no primeiro pico, e ao carbono fixo no segundo pico. Em estudos com casca de arroz nas mesmas condições, verificou-se que $99 \%$ das amostras decompunham-se até $475^{\circ} \mathrm{C}[15,16]$.

A maior parte da CA é composta por materiais orgânicos que são eliminados em baixas temperaturas (abaixo de $500{ }^{\circ} \mathrm{C}$ ). Contudo, um pequeno, mas suficiente volume de gás fica retido na matriz vítrea no início do processo de densificação a aproximadamente $600{ }^{\circ} \mathrm{C}$, quando a viscosidade é adequada para a retenção deste, o que resulta em elevadas porosidades em temperaturas mais altas. Além disso, a quantidade de carbono presente na CA também auxilia na formação da estrutura porosa devido ao seu comportamento expansivo (formação de gases) entre 650 e $1000{ }^{\circ} \mathrm{C}$ [28].

Baseado no comportamento térmico do VG e da CA, compactos com diferentes teores de VG (entre 75 e $97 \%$ em massa) e CA ( 3 a $25 \%$ em massa) foram preparados e queimados em diferentes temperaturas $\left(700\right.$ a $\left.950{ }^{\circ} \mathrm{C}\right) \mathrm{com}$ um patamar de $60 \mathrm{~min}$. A Figura 2 apresenta resultados referentes a retração diametral dos compactos (relação entre o diâmetro antes e após a queima), nos quais as identificações correspondem ao percentual de casca de arroz utilizado, por exemplo, a formulação CA03 possui $3 \%$ de casca de arroz e $97 \%$ de vidros de garrafas.

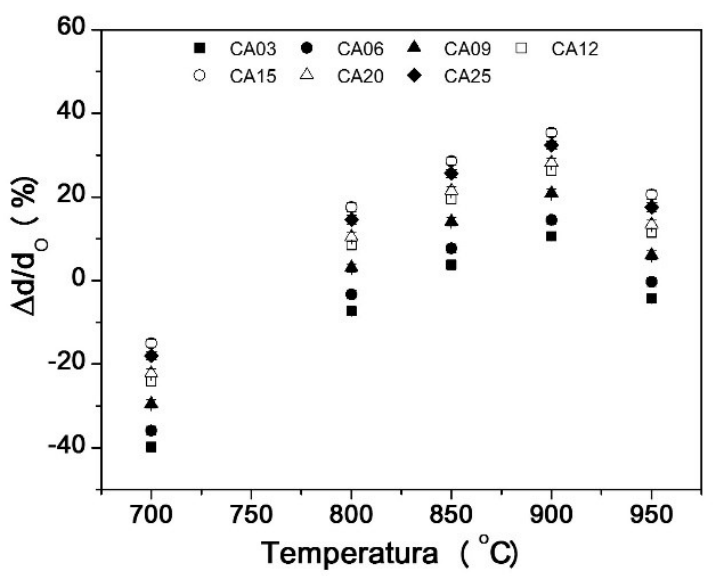

Figura 2: Valores de retração diametral das espumas contendo diferentes teores de VG (75 a 97\%) e CA (3 a 25\%) queimadas entre 700 e $950^{\circ} \mathrm{C}$ com um patamar de $60 \mathrm{~min}$.

Como pode ser observado, a partir da análise da Figura 2, todas as espumas obtidas, sem exceção, expandem no intervalo entre 700 e $900{ }^{\circ} \mathrm{C}$ e apresentam diminuição da expansão (processo inverso) entre 900 e $950{ }^{\circ} \mathrm{C}$ devido à proximidade da temperatura de fusão dos materiais. Também é possível observar que, à medida que a quantidade de CA aumenta de 3 para $15 \%$, a expansão aumenta continuamente. Contudo, para teores de CA de 20 a $25 \%$ a expansão apresenta valores situados entre 
aqueles apresentados pelas composições com 12 e 15\%. Estes comportamentos (dependência da temperatura e da quantidade do agente porogênico) são provavelmente relacionados ao aumento do volume de gases gerados pela decomposição da CA que resulta no aumento da pressão interna dos compactos/espumas, levando à ruptura das paredes dos poros, permitindo assim o escape dos gases gerados. Estes resultados indicam que a massa de CA que é capaz de promover a maior porosidade nos compactos corresponde a $15 \%$ de casca de arroz. Assim, esta formulação foi utilizada na obtenção das espumas consideradas, em continuação, neste trabalho.

A Figura 3 mostra um difratograma de raios-X de uma amostra de espuma vítrea queimada a 900 ${ }^{\circ} \mathrm{C}$ por $90 \mathrm{~min}$. De acordo com o difratograma obtido formou-se, durante a queima das espumas, cristobalita (JCPDS-01.076-0939) e silicato sódico-cálcico (JCPDS-00.023-0671). Por meio de análises quantitativas relativas, estima-se que a cristalinidade das espumas obtidas (queimadas a $900^{\circ} \mathrm{C}$ por 90 min) é da ordem de $50 \%$. A cristobalita é uma fase polimórfica do quartzo e é geralmente observada em vidros sódico-cálcicos que sofreram devitrificação. A transformação da fase cristobalita $\alpha$ para a forma $\beta$ ocorre a $200{ }^{\circ} \mathrm{C}$ e é acompanhada de grande variação de volume, o que geralmente compromete a resistência mecânica devido à formação de micro-trincas [5] nos materiais. Apesar disto, não foram observadas micro-trincas nas espumas vítreas obtidas. Este resultado indica que a espuma obtida é vitrocristalina e não vítrea.

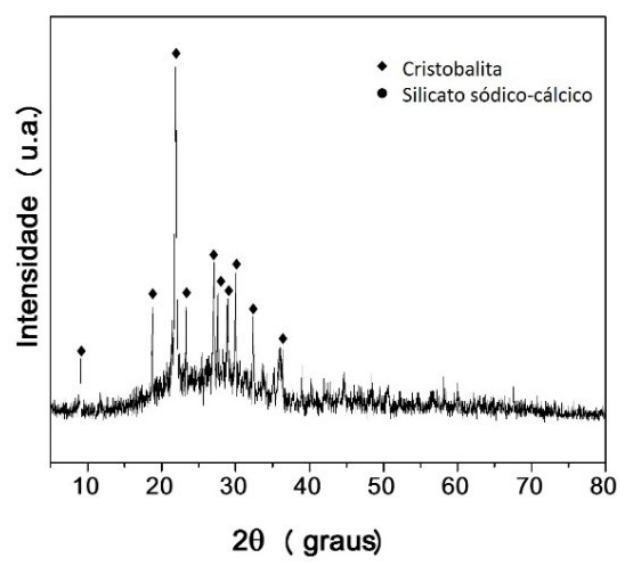

Figura 3: Difratograma de raios- $\mathrm{X}$ de amostra de espuma vitrocristalina com $15 \%$ de $\mathrm{CA}$ queimada a $900{ }^{\circ} \mathrm{C}$ por 90 $\min$.

A Tabela 3 mostra os valores de porosidade em função da temperatura $\left(700-950{ }^{\circ} \mathrm{C}\right)$ e tempo (30-120 min) de queima de compactos/espumas vitrocristalinas contendo $85 \%$ de VG e $15 \%$ de CA.

Tabela 3: Efeito da temperatura de queima e do tempo de patamar na porosidade de espumas vitrocristalinas com $85 \%$ de $\mathrm{VG}$ e $15 \%$ de $\mathrm{CA}$.

\begin{tabular}{|c|c|c|c|c|c|}
\hline \multirow{2}{*}{$\begin{array}{c}\text { Tempo de } \\
\text { patamar }\end{array}\left(\begin{array}{c}\text { Temperatura de queima }\left({ }^{\mathbf{0}} \mathbf{C}\right) \\
\end{array}\right.$} & $\mathbf{7 0 0}$ & $\mathbf{8 0 0}$ & $\mathbf{8 5 0}$ & $\mathbf{9 0 0}$ & $\mathbf{9 5 0}$ \\
\cline { 2 - 6 } & \multicolumn{5}{|c|}{ Porosidade, $\mathbf{E}(\mathbf{\%})$} \\
\hline $\mathbf{3 0}$ & $20,3 \pm 2,2$ & $76,1 \pm 0,1$ & $79,6 \pm 0,4$ & $80,2 \pm 0,8$ & $83,6 \pm 0,7$ \\
\hline $\mathbf{6 0}$ & $30,1 \pm 1,4$ & $80,6 \pm 0,3$ & $81,3 \pm 0,3$ & $82,3 \pm 0,3$ & $78,9 \pm 0,44$ \\
\hline $\mathbf{9 0}$ & $48,2 \pm 0,7$ & $79,7 \pm 0,1$ & $82,0 \pm 0,4$ & $83,5 \pm 0,2$ & $81,4 \pm 0,4$ \\
\hline $\mathbf{1 2 0}$ & $47,6 \pm 2,1$ & $78,7 \pm 0,2$ & $83,0 \pm 0,3$ & $84,3 \pm 0,2$ & $82,2 \pm 1,0$ \\
\hline
\end{tabular}

Conforme verificado na Tabela 3, o comportamento das espumas vitrocristalinas obtidas com $15 \%$ de CA segue a tendência observada na Figura 2, isto é, a porosidade aumenta rapidamente entre 700 e $800{ }^{\circ} \mathrm{C}$ (aumentando de $20 \%$ para $80 \%$ ) e, em menor proporção entre 800 e $950{ }^{\circ} \mathrm{C}$ (variando entre 80 e $84 \%$, aproximadamente).

A Figura 4 mostra os intervalos de diâmetros de poros e seus valores outliers para as espumas vitrocristalinas contendo $15 \%$ de $\mathrm{CA}$, queimadas a diferentes temperaturas $\left(800-950{ }^{\circ} \mathrm{C}\right)$ e tempos $(30$ $120 \mathrm{~min})$. 

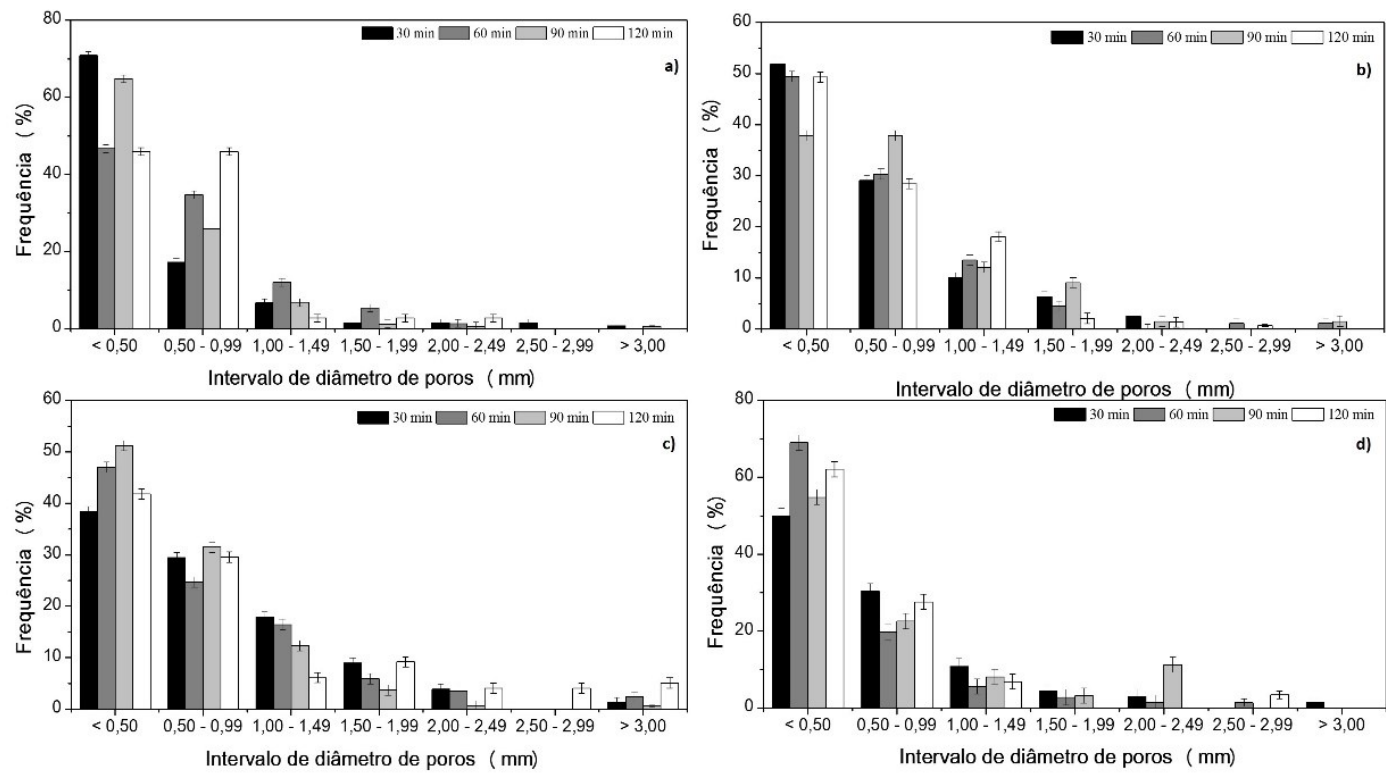

Figura 4: Diâmetros de poros em função do tempo de patamar de espumas vitrocristalinas com $15 \%$ de CA queimadas a: a) $800{ }^{\circ} \mathrm{C}$, b) $850{ }^{\circ} \mathrm{C}$, c) $900{ }^{\circ} \mathrm{C}$ e d) $950{ }^{\circ} \mathrm{C}$.

De acordo com a Figura 4 , a distribuição de diâmetros de poros das espumas vitrocristalinas obtidas em diferentes temperaturas e tempos, foi muito similar, isto é, a maior fração dos diâmetros de poros apresentou diâmetros menores que $1 \mathrm{~mm}$ e a fração de poros com diâmetros superiores a $3 \mathrm{~mm}$ não é muito significativa (a maior fração foi verificada em amostras queimadas a $900{ }^{\circ} \mathrm{C}$ ). A média dos diâmetros de poros obtidos, conforme Figura 4, variou entre $0,6 \pm 0,1 \mathrm{~mm}$ (espumas queimadas a $950{ }^{\circ} \mathrm{C}$ por $60 \mathrm{~min}$ ) e 1,0 $\pm 0,2 \mathrm{~mm}$ (espumas queimadas a $900^{\circ} \mathrm{C}$ por $120 \mathrm{~min}$ ). Na Figura 4 também é possível verificar a distribuição de tamanho dos poros, por exemplo, a $800{ }^{\circ} \mathrm{C}$ (Figura $4 \mathrm{a}$ ): $25 \%$ dos poros obtidos nas espumas vítreas queimadas por $30 \mathrm{~min}$ apresentaram diâmetros inferiores a $0,2 \mathrm{~mm}$ e $75 \%$ dos poros apresentaram diâmetros inferiores a $0,6 \mathrm{~mm}$.

Os valores médios de diâmetro de poros obtidos para as queimas em $800{ }^{\circ} \mathrm{C}$ foram variaram entre $0,5 \pm 0,1 \mathrm{~mm}$ e $0,6 \pm 0,1 \mathrm{~mm}$. Nas queimas a $850{ }^{\circ} \mathrm{C}$ os diâmetros médios dos poros obtidos apresentaram valores entre $0,7 \pm 0,1 \mathrm{~mm}$ e $0,8 \pm 0,1 \mathrm{~mm}$. As espumas obtidas com as queimas a $900{ }^{\circ} \mathrm{C}$ apresentaram diâmetros médios de $0,7 \pm 0,2 \mathrm{~mm}, 0,8 \pm 0,1 \mathrm{~mm}, 0,6 \pm 0,1 \mathrm{~mm}$ e $1,0 \pm 0,2 \mathrm{~mm}$ para os patamares de 30, 60, 90 e $120 \mathrm{~min}$, respectivamente. Os valores médios obtidos com as queimas e $950{ }^{\circ} \mathrm{C}$ encontram-se no intervalo entre $0,5 \pm 0,1 \mathrm{~mm}$ e $0,7 \pm 0,2 \mathrm{~mm}$.

Com esta análise também é possível obter os valores médios dos poros originados nas espumas com diferentes tempos de queima a diferentes temperaturas: a $800{ }^{\circ} \mathrm{C}, 25 \%$ dos poros são menores do que $0,3 \mathrm{~mm}$ e $75 \%$ são menores que $0,7 \mathrm{~mm}$; a $850{ }^{\circ} \mathrm{C}, 25 \%$ dos poros são inferiores a $0,3 \mathrm{~mm}$ e $75 \%$ são menores do que $0,9 \mathrm{~mm}$; a $900{ }^{\circ} \mathrm{C}, 25 \%$ dos poros possuem diâmetros menores que $0,3 \mathrm{~mm}$ e $75 \%$ são inferiores a 1,1 mm. Esta última condição de queima corresponde aos maiores diâmetros de poros, pois a $950{ }^{\circ} \mathrm{C}, 25 \%$ dos poros são menores do que $0,2 \mathrm{~mm}$ e $75 \%$ são inferiores a $0,8 \mathrm{~mm}$.

Todas as espumas vitrocristalinas obtidas apresentaram macroporos (ou seja, poros com diâmetros superiores a $50 \mathrm{~nm}$ ) com uma distribuição de poros relativamente bem definidas entre poros pequenos e grandes [3]. Esta heterogeneidade está associada às diferenças entre os tamanhos dos pós de CA $\left(d_{10}=7,5 \mu \mathrm{m}, d_{50}=71,0 \mu \mathrm{m}\right.$ e $\left.d_{90}=358 \mu \mathrm{m}\right)$ e VG $\left(d_{10}=1,1 \mu \mathrm{m}, d_{50}=2,3 \mu \mathrm{m}\right.$ e $\left.d_{90}=15,1 \mu \mathrm{m}\right)$ utilizados, além da diferença entre suas densidades reais (valores obtidos por picnometria, na qual a densidade real do pó de VG foi de $2,5 \mathrm{~g} / \mathrm{cm}^{3}$ e do pó de CA foi de $1,5 \mathrm{~g} / \mathrm{cm}^{3}$ ) [30].

Todas as espumas apresentaram porosidade predominantemente fechada e bem distribuída e sem fissuras, como pode ser visto nas micrografias da Figura 5. 


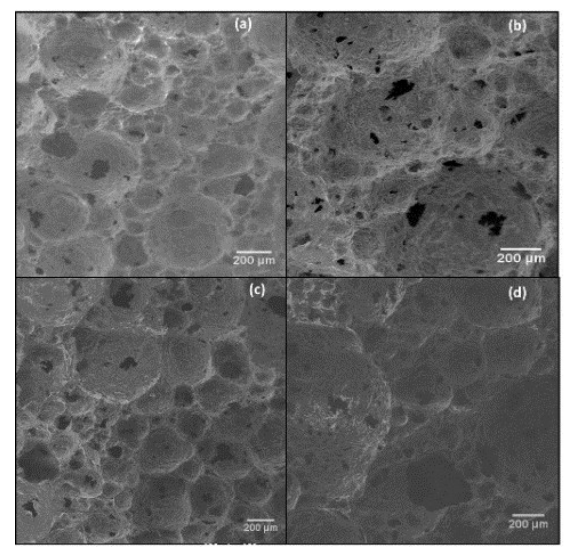

Figura 5: Micrografias (MEV) das espumas vitrocristalinas com $15 \%$ de $\mathrm{CA}$ queimadas a $900{ }^{\circ} \mathrm{C}$ por: a) $30 \mathrm{~min}$; b) $60 \mathrm{~min}$; c) $90 \mathrm{~min}$; d) $120 \mathrm{~min}$.

A Figura 6 mostra os resultados de resistência mecânica à compressão de amostras de espumas vitrocristalinas queimadas entre 800 e $950{ }^{\circ} \mathrm{C}$ por tempos entre 30 e $120 \mathrm{~min}$, sendo estes resultados em função da porosidade.

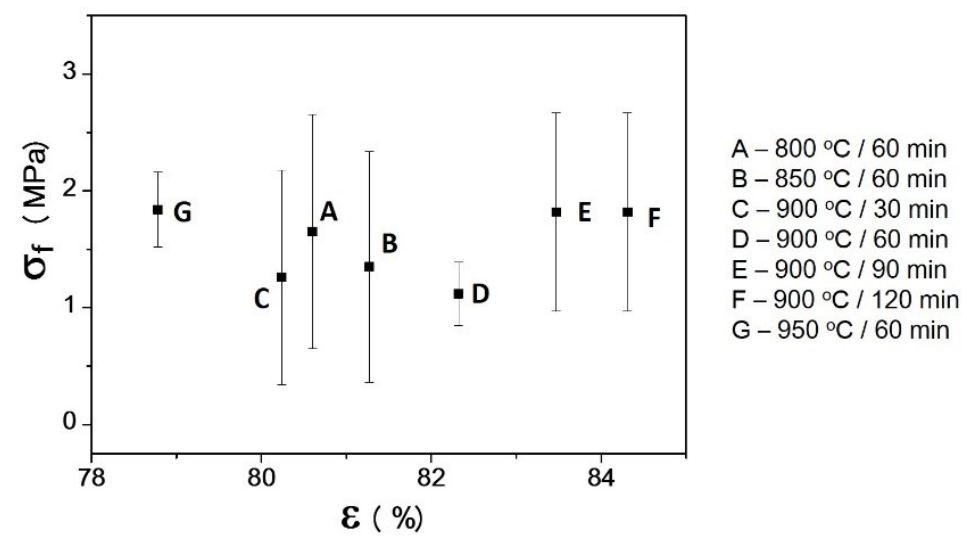

Figura 6: Resistência mecânica à compressão $\left(\sigma_{c}\right)$ em função da porosidade $(\varepsilon)$ de espumas vitrocristalinas com $15 \%$ de CA queimadas em diferentes temperaturas $\left(800-950^{\circ} \mathrm{C}\right)$ e tempos de patamar $(30-120 \mathrm{~min})$.

Na Figura 6, verifica-se que a resistência mecânica à compressão das espumas vitrocristalinas queimadas entre 800 e $950{ }^{\circ} \mathrm{C}$, por 30 a $120 \mathrm{~min}$, considerando a dispersão em torno dos valores médios, é praticamente constante (variando de 1,1 a 1,9 MPa), com uma pequena variação da porosidade (entre 80 e $84 \%$

Nas espumas com porosidades semelhantes, como aquelas queimadas a $800{ }^{\circ} \mathrm{C}$ por $60 \mathrm{~min}$ (ponto A da Figura 6) e $900{ }^{\circ} \mathrm{C}$ por 30 min (ponto C da Figura 6), pode-se perceber a influência do tamanho médio dos poros na resistência mecânica, isto é, a espuma com maior tamanho médio de poros (espuma queimada a $900{ }^{\circ} \mathrm{C}$ por $30 \mathrm{~min}$ ) apresentou menor resistência mecânica $(1,3 \pm 0,9 \mathrm{MPa})$ quando comparada àquela $(1,7 \pm 0,5 \mathrm{MPa})$ queimada a $800{ }^{\circ} \mathrm{C}$ por $60 \mathrm{~min}$. Assim, é possível perceber claramente que a porosidade e o tamanho médio dos poros exercem uma influência significativa sobre a condutividade térmica e a resistência mecânica dos sólidos celulares como também foi reportado para espumas de mesma natureza química e física [2].

A Figura 7 apresenta os resultados de condutividade térmica das espumas vitrocristalinas queimadas entre 700 e $950{ }^{\circ} \mathrm{C}$ por 30 a 120 min em função da porosidade. 


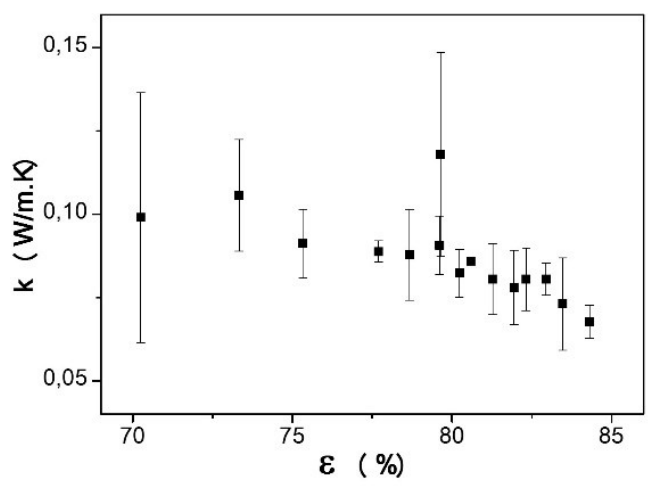

Figura 7: Condutividade térmica $(k)$ em função da porosidade $(\mathcal{E})$ de espumas vitrocristalinas com $15 \%$ de CA queimadas em diferentes temperaturas $\left(800-950^{\circ} \mathrm{C}\right)$ e tempos de patamar $(30-120 \mathrm{~min})$.

A condutividade térmica, de maneira geral, diminui rapidamente, isto é, de $1 \mathrm{~W} / \mathrm{m} . \mathrm{K}$ (condutividade térmica de um vidro sódico-cálcico sem porosidade), para valores compreendidos entre $0,15 \pm 0,06$ e $0,18 \pm 0,02 \mathrm{~W} / \mathrm{m} . \mathrm{K}$ para espumas queimadas a $700{ }^{\circ} \mathrm{C}$ em tempos de 30 a $120 \mathrm{~min}$ com porosidades entre 20 e $48 \%$ [25]. Com o aumento da porosidade para valores máximos de até $85 \%$, conforme verificado na Figura 7, a condutividade térmica das espumas é cerca de 0,07 $\pm 0,01 \mathrm{~W} / \mathrm{m} . \mathrm{K}$.

Analisando especificamente todos os valores de porosidade e condutividade térmica obtidos a partir de medidas realizadas em amostras de espumas queimadas com diferentes ciclos de queima, é possível concluir que os menores valores médios de condutividade térmica $(0,07 \pm 0,01$ a $0,09 \pm 0,02$ $\mathrm{W} / \mathrm{m} . \mathrm{K}$ ) foram obtidos em espumas queimadas entre 800 e $900{ }^{\circ} \mathrm{C}$ por 30 a $120 \mathrm{~min}$. O tempo de patamar não influenciou de maneira significativa na condutividade térmica das espumas. Contudo, a temperatura de queima apresentou maior efeito, pois promoveu o aumento da porosidade e, também, variação do tamanho dos poros. De fato, as espumas com menor porosidade foram aquelas queimadas a $700{ }^{\circ} \mathrm{C}$ que apresentaram, também, os maiores valores de condutividade térmica.

Os valores de condutividade térmica e de resistência mecânica determinados em amostras das espumas vitrocristalinas produzidas neste trabalho, exceto para àquelas queimadas a $700{ }^{\circ} \mathrm{C}$ cuja porosidade é relativamente pequena são compatíveis com aqueles de espumas vítreas comerciais (material de comparação) que costumam apresentar resistência mecânica à compressão entre 0,4 e $6 \mathrm{MPa}$ e condutividade térmica entre 0,04 a $0,08 \mathrm{~W} / \mathrm{m} . \mathrm{K}[1,2]$.

\section{CONCLUSÕES}

Os resultados evidenciaram a possibilidade de produção de espumas vitrocristalinas (isolantes térmicos) a partir de composições otimizadas contendo $85 \%$ de vidros de garrafas (VG) e $15 \%$ de casca de arroz (CA), como agente porogênico, com diâmetros médios de poros de $0,71 \mathrm{~mm}$ a partir de casca de arroz com $\mathrm{d}_{50}=71 \mu \mathrm{m}$. As melhores combinações de porosidade, condutividade térmica e resistência mecânica foram obtidas com as espumas vítreas queimadas a $900{ }^{\circ} \mathrm{C}$ por 30 a $120 \mathrm{~min}$. Neste caso, a porosidade variou entre 80 e $84 \%$ enquanto a condutividade térmica e a resistência mecânica à compressão variaram entre 0,068 e $0,082 \mathrm{~W} / \mathrm{m} . \mathrm{K}$ e entre 1,1 e $1,9 \mathrm{MPa}$, respectivamente.

Além disto, os resultados indicaram que as espumas vitrocristalinas obtidas são fortes candidatas em aplicações que envolvam sistemas de isolamento térmico já que apresentam características e propriedades semelhantes às dos produtos comerciais de mesma natureza química e física.

\section{AGRADECIMENTOS}

Os autores agradecem a FAPESC/CNPq (PRONEX T.O. N 17431/2011-9).

\section{BIBLIOGRAFIA}

[1] BERNARDO, E., CEDRO, R., FLOREAN, M., et al., "Reutilization and stabilization of wastes by the production of glass foams”, Ceramics International, v. 33, pp. 963-968, 2007. 
[2] SCheffler, M., COLOMBO, P., Cellular Ceramics: Structure, Manufacturing, Properties and Applications, Weinheim, WILEY-VCH Verlag GmbH \& Co. KGaA, 2005.

[3] ROUQUEROL, J., AVNIR, D., FAIRBRIDGE, C.W., et al., "Recommendation for the characterization of porous solids", Pure \& Applied Chemistry, v. 66, pp. 1739-1758, 1994.

[4] ZHU, X., JIANG, D., TAN, S., "Preparation of silicon carbide reticulated porous ceramics", Materials Science and Engenieering, v. 323, pp. 232-238, 2002.

[5] TAURINO, R., LANCELLOTI, I., BARBIERI, L., et al., "Glass-ceramic foams from borosilicate glass waste", International Journal of Applied Glass Science, v. 5, pp. 136-145, 2014.

[6] STUDART, A.R., GONZENBACH, U.T., TERVOORT, E., et al., "Processing routes to macroporous ceramics: A review", Journal of the American Ceramic Society, v. 89, pp. 1771-1789, 2006.

[7] ALVES, O.L., GIMENEZ, I.F., MAZALI, I.O., Vidros. Química Nova na Escola, São Paulo, Brasil, v. maio, n. Ed. Esp., p. 13-24, 2001.

[8] DIAS, G.M.V., ARCARO, S., CESCONETO, F.R., et al., "Production and characterization of glass foams for thermal insulation", Chemical Engineering Transaction, v. 43, pp. 1777-1782, 2015.

[9] ZILLI, M., ARCARO, S., CESCONETO, F.R., et al., "Production and characterization of ceramic foams from industrial solid waste", Chemical Engineering Transaction, v. 43, pp. 1783-17882, 2015.

[10] MUGONI, C., MONTORSI, M., SILIGARDI, C., et al., "Design of glass foams with low environmental impact", Ceramics International, v. 41, pp. 3400-3408, 2015.

[11] FERNANDES, H.R., FERREIRA, D., ANDREOLA, F., et al., "Environmental friendly management of CRT glass by foaming with waste egg shells", Ceramics International, v. 40, pp. 13371-13379, 2014.

[12] NAGRALE, S.D, HAJARE H., MODAK P.R., "Utilization of rice husk ash", International Journal of Engineering Research and Applications, v.2, n.4, pp.1-5, 2012.

[13] CONAB - COMPANHIA NACIONAL DE ABASTECIMENTO, Levantamento de Safra 2014 http://www.conab.gov.br/ .Acessado em julho de 2016.

[14] KRISHNARAO, R.V, SUBRAHMANYAM, J., KUMAR, T.J., "Studies on the formation of black particles in rice husk silica ash", Journal of European Ceramic Society, v.21, pp.99-104, 2001.

[15] KOK, M.V., ÖZGÜR, E., "Thermal analysis and kinetics of biomass Samples", Fuel Processing Technology, v. 106, pp. 739-743, 2013.

[16] MHILU, C.F., "Analysis of energy characteristics of rice and coffee husks blends", ISRN Chemical Engineering, v.2014, pp. 1-6, 2014.

[17] ASTM - Annual book of ASTM standards: E 871 - 82. Standard test method moisture analysis of particulate wood fuels, 2006.

[18] ASTM - Annual book of ASTM standards: E 872 - 82. Standard test method for volatile matter in the analysis of particulate wood fuels, 2006.

[19] ASTM - Annual book of ASTM standards: E 1755 - 01. Standard test method for ash in biomass, 2007.

[20] ASTM - Annual book of ASTM standards: E 1756 - 08. Standard test method for determination of total solids in biomass, 2008.

[21] GARCÍA, R., PIZARRO, C., LAVÍN, A.G., et al., "Characterization of Spanish biomass waste for energy use", Bioresource Technology, v. 103, p. 249-258, 2012.

[22] NOGUEIRA, G., Combustão e gasificação de biomassa sólida, Brasília: Ministério de Minas e Energia, 2008.

[23] ASTM - Annual book of ASTM standards: D3576-98. Standard test method for cell size in rigid cellular plastics, 1998.

[24] NAVARRO, J.M.G., El vidrio, $3^{\mathrm{a}}$ ed, Madrid - Spain, CSIC - Consejo Superior de Investigaciones Científicas, 2003.

[25] PANDOLFO, C., BRAGA, H.J., SILVA V.P., et al., Atlas climatológico do Estado de Santa Catarina. Florianópolis: Epagri, 2002. 
[26] YANG, H., YAN, R., CHEN, H., et al., "Characteristics of hemicellulose, cellulose and lignin pyrolysis", Fuel, v.86, pp. 1781-1788, 2007.

[27] VIEIRA, A.C., SOUZA, S.N.M., BARICCATTI, R.A., et al., "Caracterização da casca de arroz para geração de energia", Revista Varia Scientia Agrárias, v. 3, n.1, pp. 51-57, 2013.

[28] CHI, W., JIANG, D., HUANG, Z., TAN, S., "Sintering behavior of porous SiC ceramics", Ceramics International, v. 30, pp. 869-874, 2004.

[29] ASADIERAGHI, M., WMAW, D., "Characterization of lignocellulosic biomass thermal degradation and physiochemical structure: effects of demineralization by diverse acid solutions", Energy Conversion and Management, v.82, pp.71-82, 2014.

[30] OHJI, T., FUKUSHIMA, M., "Macro-porous ceramics: processing and Properties", International Materials Reviews, v. 57, pp. 115-131, 2012. 